This is the final peer-reviewed accepted manuscript of:

Rupi, F., Bernardi, S., Rossi, G. et al. The Evaluation of Road Network Vulnerability in Mountainous Areas: A Case Study. Netw Spat Econ 15, 397-411 (2015).

The final published version is available online at: https://doi.org/10.1007/s11067-014-9260-8

Rights / License:

The terms and conditions for the reuse of this version of the manuscript are specified in the publishing policy. For all terms of use and more information see the publisher's website. 
The evaluation of road network vulnerability in mountainous areas: a case study

Federico Rupi ${ }^{1}$, Silvia Bernardi ${ }^{2}$, Guido Rossi ${ }^{3}$ and Antonio Danesi ${ }^{4}$

1 - federico.rupi@unibo.it Corresponding Author

University of Bologna, Italy, Faculty of Engineering, DICAM Dep., Transport research group;

2 - silvia.bernardi9@unibo.it

University of Bologna, Italy, Faculty of Engineering, DICAM Dep., Transport research group

3 - guido.rossi6@unibo.it

University of Bologna, Italy, Faculty of Engineering, DICAM Dep., Transport research group

4 - antonio.danesi@unibo.it

University of Bologna, Italy, Faculty of Engineering, DICAM Dep., Transport research group 


\title{
The evaluation of road network vulnerability in mountainous areas: a case study
}

\author{
Federico Rupi (*), Silvia Bernardi( (*),Guido Rossi(*) and Antonio Danesi( $\left(^{*}\right)$
}

\begin{abstract}
This paper deals with the issue of road network vulnerability, with special reference to a mountainous area case. It describes the implementation of a methodology that ranks links of a network according to their importance in maintaining a proper connectivity between all origindestination pairs. Such a ranking can be particularly helpful in prioritising maintenance investments to be planned along the links of a road network.

Following a conceptual approach observed in transport literature, we consider vulnerability to be connected to importance, i.e. a measure of the consequences of the collapse of a network element. In the present study we introduce a new definition of importance - with respect to a given link that simultaneously includes two aspects: its level of usage, i.e. how many people typically use the link when travelling in a given time window, and the impact that the closure of that link could have on the general functionality of the network as a whole.

The methodology proposed was implemented in order to obtain a ranking of importance for the links of a real-scale network, i.e. the road network of the Province of Bolzano, a highly mountainous area located in the Italian Alps.
\end{abstract}

\section{Introduction}

Increasingly over the past twenty years issues relating to the robustness and vulnerability of road networks have become the subject of considerable attention. Transport networks fully belong to the category of "lifelines", all the many services essential to society as a whole. The primary objective of this study was to identify the most vulnerable elements within a network, and so to determine a ranking of priorities in the allocation of financial resources for maintenance interventions.

This paper deals with the issue of road network vulnerability, with special reference to mountainous areas. Road network vulnerability is a major determinant of risk in transport operations and its evaluation is particularly interesting in mountainous areas, mainly for two 
reasons. Firstly, disruptions to road infrastructure occur more frequently in mountainous areas than elsewhere, due to the typically high intensity of natural events (such as debris-flows, avalanches, rock-falls) that can jeopardise network operations. Secondly, mountain road networks generally have a low level of connectivity, being more linear in configuration rather than forming a grid: this leads to the relatively high probability that connections between some areas of a transport network can be completely interrupted when one or more elements of the network cannot be used.

This paper describes the implementation of a methodology that ranks the links of a network according to their importance in maintaining a proper connectivity between all origin-destination pairs. Results can be particularly helpful in prioritising ordinary and extraordinary maintenance investments to be planned along the links of a road transport network. Indeed, the improvement of all vulnerable links of a network in order to make them able to withstand any hazardous event would entail unacceptable costs. This implies the need prioritize the allocation the limited resources, in the framework of an investment planning by part of local administrations or agencies (Matisziw et al. 2010; Du and Peeta, 2014).

The case study being examined refers to the territory under the administration of the Province of Bolzano, in the Italian Alps, following an agreement between the local authority of Bolzano and the Department of Civil Engineering of the University of Bologna to carry out this research work within the framework of the EU PARAmount project ${ }^{1}$. In practice, however, this methodology can be applied to other geographical contexts.

The paper is outlined as follows: Section 2 provides a brief conceptual framework about the definition of vulnerability and the main approaches proposed in literature. In Section 3 we present our methodological approach and the indicators of link importance. Section 4 describes the main features of the case study to which the methodology has been applied and gives the results. Conclusions are presented in Section 5.

\section{Conceptual framework}

To date, there is no a widely accepted definition of vulnerability (Berdica, 2002; Taylor and D'Este, 2003, Knoop et al., 2008, Taylor and Susilawati, 2012): there are numerous definitions proposed in literature with correspondingly numerous methodologies and indicators that try to describe and quantify the consequences of hazardous events (such as debris-flows, avalanches, rock falls, car-accidents and even natural disasters or terrorist attacks) or, more generally, of disturbances

\footnotetext{
${ }^{1}$ The PARAmount project for reliability and security of Alpine transport infrastructure related to mountainous hazards in a changing climate.
} 
into the functionality of a transport network. These disturbances occur with a certain probability and have as their primary effect a reduction in link capacity and/or a variation in demand (Sumalee and Kurauchi, 2006; Snelder et al., 2012).

Among the first studies on transport network vulnerability are those by Berdica (2002) and D'Este and Taylor (2003). Berdica defines "vulnerability" as " a susceptibility to incidents that can result in considerable reductions in road network serviceability', where serviceability of a link/route/road network is interpreted as "the possibility to use that link/route/road network during a given period".

D'Este and Taylor (2003) introduced the concept of accessibility in vulnerability assessment studies, stating that a network link is critical if loss of the link significantly diminishes the accessibility of a particular node. This approach was re-iterated by Taylor et al. (2006), and Taylor and Susilawati (2012), whose works further introduced the inverse of accessibility, remoteness.

Other well-known aspects leading back to vulnerability are robustness and reliability. Although reliability and robustness are correlated, they are not identical (Snelder et al., 2012): robustness focuses on the impact of the disturbance, while reliability focuses on the frequency of occurrence of the disturbance, or better, on its probability. According to Bell (2000) "A network is reliable if the expected trip costs are acceptable even when users are extremely pessimistic about the state of the network'. Moreover Immers et al. (2004) expressed that reliability is a user oriented quality while robustness is a characteristic of the system itself. Scott et al. (2012) define the network robustness for evaluating the critical importance of a given network link to the overall system as the change in travel-time cost associated with rerouting all traffic in the system should that link become unusable. Similarly, Yin et al. (2009) interpret the concept of robustness as the decrease of network performance due to the selected removal of links.

D'Este and Taylor (2003) has also pointed to the difference between vulnerability and reliability, where the latter is linked to probability. They claim that vulnerability is related to the effects of road disruption, without considering the probability of that disruption.

Other authors, such as Husdal (2004), Bono and Gutiérrez (2011) and Luathep et al. (2011) distinguished between the two aspects of reliability and vulnerability of a transport network. According to these authors, if reliability is a measure of network stability, vulnerability should be a measure of the consequences of a collapse (or under-functioning) of a network element. Note that Carlson et al. (2012), as well as Rose (2009), consider vulnerability as pre-disaster condition (see Reggiani et al. (2014) for a review). 
Bearing in mind the well-known risk theory which states that risk is the product of probability and effect, making a distinction between vulnerability and reliability (or probability of disruption) is a valid and accepted approach.

Jenelius et al. (2006, 2009, 2010) and Nicholson and Du (1997) follow the above approach and compare the concept of vulnerability to that of criticality for the elements of a network. Criticality is given by the combination of two concepts, weakness and importance. In other words, an element can be defined as critical if it is, at the same time, weak - i.e. the probability of its failure is high and important - i.e. the consequences of its loss are significant for the whole system.

In accordance with what has been briefly summarised, we argue that risk evaluation should be divided into one component that refers to the probability of a disruption occurring, and another that refers to the consequences resulting from such a disruption to the functioning of the network as a whole and, therefore, that studies on vulnerability should focus on this second aspect. It follows that the probability of a disruption to a network component will not be the subject of this paper, as our main aim will be to evaluate link vulnerability, which can be interpreted as a conditional vulnerability. Sarewitz et al. (2003) also point to the disadvantages of including the probability of failure in vulnerability studies, as it is particularly difficult to estimate the probability of extreme events, such as natural disasters or terrorist attacks. We claim that evaluating the probability of events occurring, especially natural events, should involve a special study, for the specific territory under examination, to be carried out by experts in the phenomena that occur in that territory itself. This assessment of probability can later be superimposed to that of vulnerability, and these two aspects combined to locate criticalities.

Following the works of Nicholson and Du (1997) and Jenelius (2006, 2009, 2010), we use "importance" to measure the consequences of a link disruption. As will be illustrated in Section 3, we consider two aspects relating to link importance. The first refers to the level of usage, since a link can, evidently, be considered as important if many users rely on it for their daily trips. The second aspect involves a wider approach to link importance, to evaluate the consequences on the entire network - in terms of increased cost for users - of the total closure of a link.

\section{Methodology}

\subsection{Link importance index}


The aim of the present study is to answer the following question: which are the most critical links for a mountainous road network? In such a perspective, analysing the vulnerability of a road network corresponds to designing a process to create a ranking of network links according to their importance. In this framework the vulnerability analysis of a road network was conducted by adapting and integrating the approach proposed by other authors (Taylor and Susilawati, 2012; Sullivan et al., 2010; Qiang and Nagurney, 2008; Chen et al., 2007; Berdica and Mattsson, 2007; Jenelius et al., 2006), based on measures of link importance, estimating the contribution of a link to the serviceability of the network. In particular, a set of relevant links can be assumed to be successively and completely - considering both directions - closed, forcing travellers to choose other less convenient routes, with an increase of the total trip cost on the network.

A significant problem resulting from the complete removal of a link in order to model linkdisruption, is the possible disconnection of the network in two distinct parts. Evidently in this case it becomes impossible to evaluate the importance of a link by only using the total cost variation, as this total cost variation becomes infinite.

One approach to address problems associated with these isolating links is to use a high percentage-based link-capacity disruption level (Sullivan et al. 2010), for example a 99\% reduction in capacity. Such an approach, however, appears to be scarcely rigorous, since the level of linkcapacity reduction is completely arbitrary.

The approach proposed by Jenelius, on the other hand, consists in measuring the importance of the isolating links (called "cut links") in terms of their unassigned demand, rather than the total-trip cost variation. The higher the unassigned demand, the higher the measure of importance of the cut link.

In the present study, the above-mentioned approach is integrated by taking into account, in addition to the impact that the closure of a link can have on the general functionality of the network as a whole, the level of that link usage, i.e. how many people typically use the link for their trips, commonly considered by technicians for addressing maintenance investments.

In this study, the following expression for the index of link importance, $L I_{j}$, has been introduced to evaluate the importance of a generic link $j$ :

$$
L I_{j}=\beta \cdot F\left(A D T_{j}\right)+(1-\beta) \cdot G\left(\Delta C_{j}\right)
$$

where $F$ is a function of $A D T_{j}$, i.e. the average daily traffic along link $j, G$ is a function of $\Delta C_{j}$, i.e. the increase in the network users' total cost due to the interruption of link $j$ (calculated with respect to ordinary undamaged network configuration) and $\beta$ is a sensitivity parameter. In this paper we 
refer to the first function, $F\left(A D T_{j}\right)$, as local importance, and to the second, $G\left(\Delta C_{j}\right)$, as global importance.

The expression of link importance defined in (1) allows the two functions for local and global importance to be weighted differently. Simply assigning two - complementary - weighting parameters, the analyst can decide which are more important: either the most used links or the most "strategic" links, in terms of network functionality. We also assume that the $\beta$ parameter range is between 0 and 1: the higher the value of $\beta$, the higher the weighting of the local importance function and therefore the reliability of the measured average daily traffic, relative to the global importance. Such a decision could be based on "political" assumptions regarding the concept of vulnerability (see Jenelius et al., 2006, for aspects of equal opportunity and social efficiency). To best assign a value for the $\beta$ parameter, analysts should also consider the reliability of all available data. If, for instance, the simulation models used to determine global importance are inaccurate, a higher weight can be assigned to the ADT data - generally less affected by accuracy issues thereby improving the overall reliability of the final evaluation of importance. In the case where $\beta$ is assumed to be equal to 0.5 , the two functions $F$ and $G$ are given the same weight in the computation of the link importance index.

In the following paragraphs 3.2 and 3.3, we will examine the local and global components of the link importance index more extensively.

\subsection{Local Importance}

In terms of the local importance function, if a high number of vehicles travel along a link, its vulnerability is higher than that of other links with less traffic. It is therefore reasonable to consider link importance as being proportional to link traffic. In order to take this into account, the local importance indicator $F_{j}$ has been introduced, defined as follows:

$$
F_{j}=F\left(A D T_{j}\right)=\frac{A D T_{j}-A D T_{\text {min }}}{A D T_{\text {max }}-A D T_{\text {min }}}
$$

where $A D T_{j}$ is the average measured daily traffic along link $j, A D T_{\min }$ and $A D T_{\max }$ are respectively the minimum and the maximum $A D T$ measured on the set of links whose $A D T$ is known.

The local importance indicator calculated in this way ranges from 0 to 1 , where 0 is the score assigned to the link in the ranking with less traffic, and 1 the score assigned to that with most traffic. It is worth noting that such an indicator is non-dimensional. 


\subsection{Global Importance}

Regarding the function of global importance, it is the variation in the generalised trip cost for all network users over a given time interval. This variation results from the assignment of transport demand to the network when the link in examination is closed. According to Qiang and Nagurney, (2008), Berdica and Mattsson (2007), Jenelius (2006), Sullivan (2010), the importance of a link, in terms of network functionality, is proportional to the increase in the overall network trip cost due to the removal of the link itself. Thus, the first step to evaluate the global importance function consists in evaluating $\Delta C_{j}$, i.e. the total variation in trip cost for the network caused by the closure of a generic link $j$ :

$$
\Delta C_{j}=C_{j}-C_{0}, \quad \forall j \in B
$$

where $C_{j}$ is the total cost of the network assuming link $j$ to be interrupted (damaged network conditions), $C_{0}$ is the total cost of the network calculated when all links in the network function normally (undamaged network condition) and $B$ is the set of the relevant links being studied where the average daily traffic is known. The total trip $\operatorname{cost} C$ for the network is the sum of all generalised trip costs paid by all users, and is obtained as:

$$
C=\sum_{\forall O D} \sum_{\forall k \in I_{O D}} C_{k} \cdot V_{k}
$$

where $C_{k}$ is the generalised cost and $V_{k}$ is the simulated volume of a generic route $k$ between the generic origin $O$ and the generic destination $D$, and $I_{O D}$ is the set of existing routes connecting $O$ to D.

Considering function (3), in order to calculate the total trip cost variation $\Delta C_{j}$, the assignment of the demand to the network must be carried out first for the undamaged condition (obtaining $C_{0}$ ), and then for all the $j^{\text {th }}$ damaged conditions (where link $j$ is missing).

In brief, a procedure to estimate the increase in total cost for a damaged network calls for the following steps:

1. the undamaged network is simulated, and both traffic flows and the generalized trip costs are estimated, so that the total cost for the undamaged network can be calculated, this being the cost borne by all network users completing their trips; 
2. link $j$ is completely interrupted in both directions and, as a result, a new model is obtained representing the damaged network without the bi-directional link $j$;

3. the $j^{\text {th }}$ damaged network is simulated, leading to the calculation of the total-trip costs for this network; moreover, if the closure of link $j$ determines the isolation of part of the network, in addition to total-trip cost, the unassigned demand is calculated;

4. steps 2 and 3 are repeated for all the links included in the set $B$ of links whose importance is to be calculated.

As will be illustrated in Section 4, an automated procedure to apply these steps was introduced using simulation software.

Depending on the topology of the network, when dealing with real-scale networks, the closure of a certain link can cause a disconnection between two parts of the network, leading to some centroids remaining isolated. This happens when the topology of the network does not allow for any re-routing for a given O-D pair. These links that, if closed, determine this disconnection of the network are called "cut links" (Jenelius, 2006). It is worth noting that cut links occur more often in road networks in mountain areas. Mountainous road networks generally have a low level of connectivity, as they are almost linear and can rarely form a grid. This leads to the relatively high probability that connections between some origin-destination pairs are completely interrupted when one or more elements are no longer functioning.

Evidently, in case where link $j$ is a cut link, the total cost variation $\Delta C_{j}$, as calculated above in order to determine the global importance of the link, assumes an infinite value - since the travel time becomes infinite for part of the trips. In this case, $D_{O D}^{j}$ is the demand from origin $O$ to destination $D$ that cannot be satisfied link $j$ is closed, and there is no connection between the $O-D$ pair. The higher the unsatisfied demand, the higher the reduction in accessibility caused by the closure of a cut link.

It should be noted that one of the aims of this study is to obtain a clear ranking of all links in terms of their importance, and includes both cut and non-cut links. In order to define this ranking and in line with the "political" considerations made by Jenelius, we start from the assumption that cut links can be seen as more important than non-cut links in the global network perspective: their closure causes not only an extra cost to network users but the isolation of some of them, who find no way to complete their trip.

Considering the presence of cut links, the global importance index of a link for the operation of the road network as a whole, $g_{j}$, can be computed as: 


$$
g_{j}=\left\{\begin{array}{c}
\Delta C_{j}, \quad \forall j \in B \quad D_{O D}^{j}=0 \\
\Delta C_{j}+\alpha \cdot D_{O D}^{j}, \quad \forall j \in B \quad D_{O D}^{j} \neq 0
\end{array}\right\}
$$

where $\alpha$ is equal to the value of a missing trip between the generic origin $O$ and destination $D$, due to the closure of link $j$. Such a formulation assures that a higher measure of global importance is associated to cut links. Among cut links, the higher the unsatisfied demand resulting from their closure, the higher the global importance measure obtained from (5).

Since the objective is to combine global importance with local importance as shown in (1), above indicator $g_{j}(5)$ must be normalised as follows:

$$
G_{j}=\frac{g_{j}-g_{\min }}{g_{\max }-g_{\min }}
$$

where $g_{\min }$ and $g_{\max }$ are, respectively, the minimum and the maximum $g_{j}$ obtained from the set of links under study.

The indicator $G_{j}$ thus defined ranges between 0 and 1 , where 0 the score assigned to the least global important link in the ranking, and 1 the score assigned to the most important.

Once the two components have been calculated as illustrated, i.e. local importance score and global importance score, they can be combined as in (1) to determine link importance.

\section{Case study}

The methodology proposed in the previous section was implemented to obtain a ranking of importance for the links of a real-scale network, i.e. the road network of the area of Bolzano, an autonomous province in northern Italy (Fig. 1).

The road network of Bolzano serves a mountainous area, located entirely in the Alps, with a population of about 511,750 and attracting thousands of visitors every year. Covering an area of almost $7,400 \mathrm{~km}^{2}$, it is the largest province in Italy. Table 1 gives data about the annual incoming tourists and the average daily traffic.

From north to south, it is crossed by a motorway, the A22, and several national roads, among which the SS12, SS38, SS42, SS49, SS238 and SS621. 


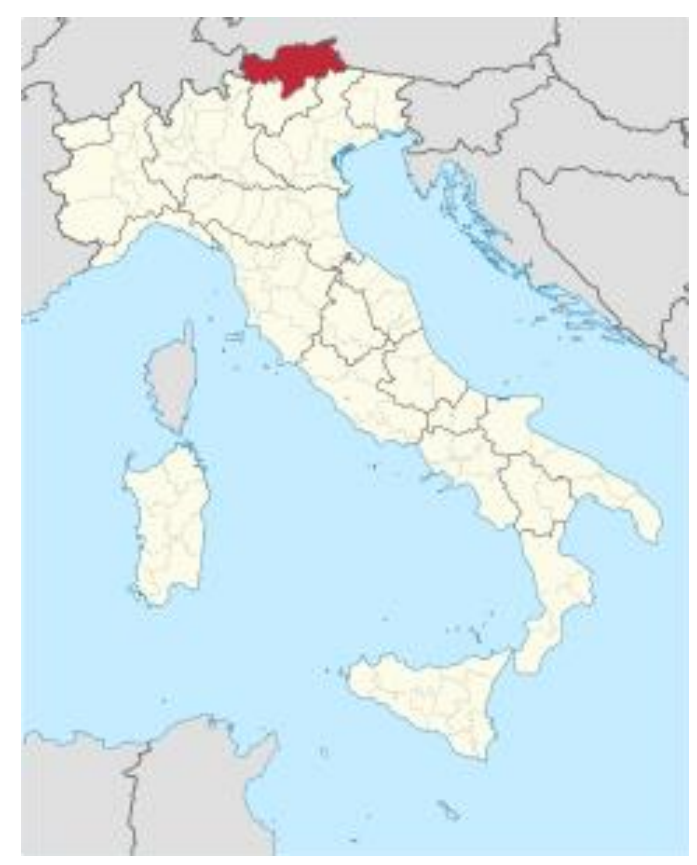

Figure 1 -Province of Bolzano in northern Italy.

Table 1. Arrivals by Country and Average Daily Traffic in the Province of Bolzano, for the year 2013.

\begin{tabular}{|c|c|c|c|}
\hline Country of origin & Arrivals (person/ year) & Traffic Type & ADT (vehicles/year) \\
\hline Italy & $2,109,867$ & Summer & 707,514 \\
\hline Germany & $2,702,795$ & Winter & 591,294 \\
\hline Austria & 271,694 & Day & 559,074 \\
\hline Switzerland and Liechtenstein & 302,830 & Night & 90,807 \\
\hline Benelux & 186,829 & People & 585,657 \\
\hline Other Countries & 467,566 & Freight & 63,659 \\
\hline \multirow[t]{2}{*}{ Total } & $6,041,581$ & Light & 612,526 \\
\hline & & Heavy & 36,790 \\
\hline
\end{tabular}




\subsection{Input models: network and O/D matrix}

The network model includes all roads except local roads and is composed of 1,607 nodes (of which 293 represent centroids, 726 junctions, and 588 represent a change in the road geometric features) and 3,500 bi-directional links (Fig.2). The link importance analysis was carried out for a sub-set of 2,158 links, where $A D T$ values have been measured and made available by the Bolzano administration.

As already noted, road networks serving mountainous areas often have a significant number of cut links. The topology of the network is such that certain areas are served by a single road, which means that, if part of this road is disrupted, there is no valid rerouting alternative and part of the demand remains unsatisfied. For this network, in particular, about $40 \%$ of the links are cut links.

In terms of the link cost function, link travel time $t_{j}$, expressed in minutes, has been defined using the formulation proposed by the Bureau of Public Roads (1964):

$$
t_{j}=t_{0, j} \cdot\left[1+\gamma\left(\frac{f_{j}}{C_{j}}\right)^{\delta}\right]
$$

where $t_{0, j}$ is the link travel time in free-flow conditions, $C_{i}$ is the link capacity $(\mathrm{veh} / \mathrm{h}), f_{i}$ is the simulated flows (veh/h); $\gamma$ and $\delta$ are calibration coefficients specified on the basis of the road type.

Regarding the demand model, the $O-D$ matrix was provided by the Bolzano administration, and relates to the week-day morning peak-period, referring to several years ago; it gives the demand between the 293 centroid nodes included in the network model. Every internal centroid corresponds on average to an area of $26 \mathrm{~km}^{2}$ with a population of about 1800. This matrix has been updated using information about more recent traffic counts along a set of links. 


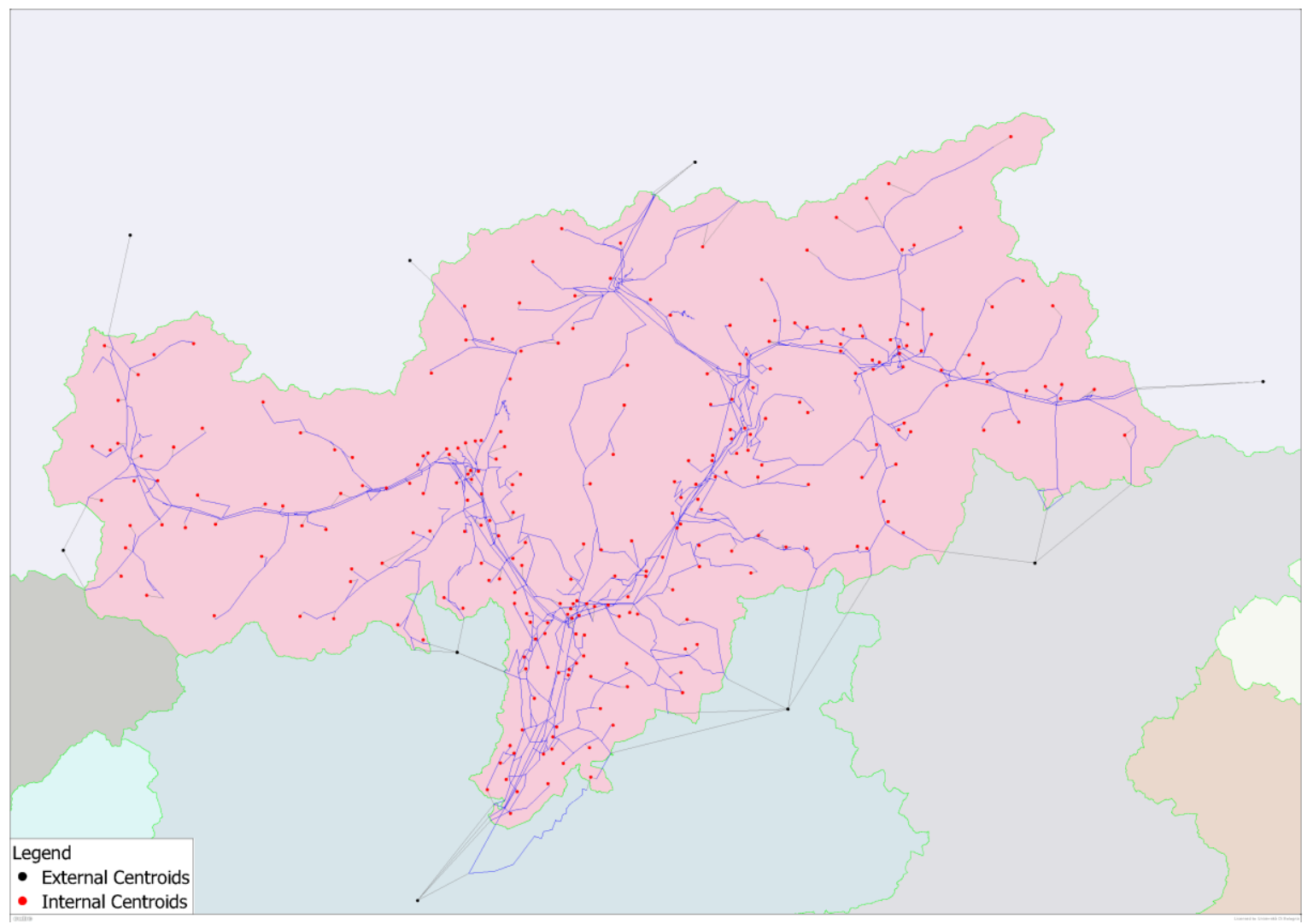

Figure 2 - The road network of Bolzano

\subsection{Simulation and results}

The methodology to determine the importance of the network links, as illustrated in Section 3, was implemented for the Bolzano area network, under the assumption that $\beta$ is equal to 0.5 (same weight for local and global importance). As mentioned above, in order to calculate the total-trip cost of the network for each damaged scenario - each one considering the removal of a link - as well as the eventual unassigned demand, the simulation was run using a commercial software, Cube by Citilabs. Once the network and demand models are imported, the software elaborates a specific script for the assignment procedure. A Deterministic User Equilibrium (DUE) model was adopted for the assignment. DUE assignment is obtained by applying the equilibrium approach for congested networks under the assumption of deterministic path choice behaviour. In the case of congested networks, the resultant path (or link) flows correspond to the condition expressed by Wardrop's first principle: "For each O-D pair the path equilibrium cost used is equal, and is less than or equal to the equilibrium cost of each unused path" (Cascetta, 2008).

Going into more detail, link flows have been first simulated for the base scenario (undamaged network) and the model corroborated by comparing the simulated flows with those measured, and 
computing the Root Mean Square Error. This meant that the total-trip cost for the undamaged network could be determined using formula (4). The software then allowed us to re-run the assignment procedure, each time removing a bi-directional link $j$, calculating link flows and totaltrip cost for the damaged network; in the case where the link $j$ was a cut link, the software also provided the unassigned demand.

Finally, the measure of importance for each link was computed using the formulations described in Section 3 (1), (2), (5) and (6). In this application, we assumed that the value of $\alpha$ is such that the top values of $g_{j}$ are all associated to cut links. As has already been noted, this assumption corresponds to the specific "political" choice of attributing the highest global importance to a missing connection. On completion of the procedure, we obtained the desired ranking for all the links of the network, in order of decreasing importance.

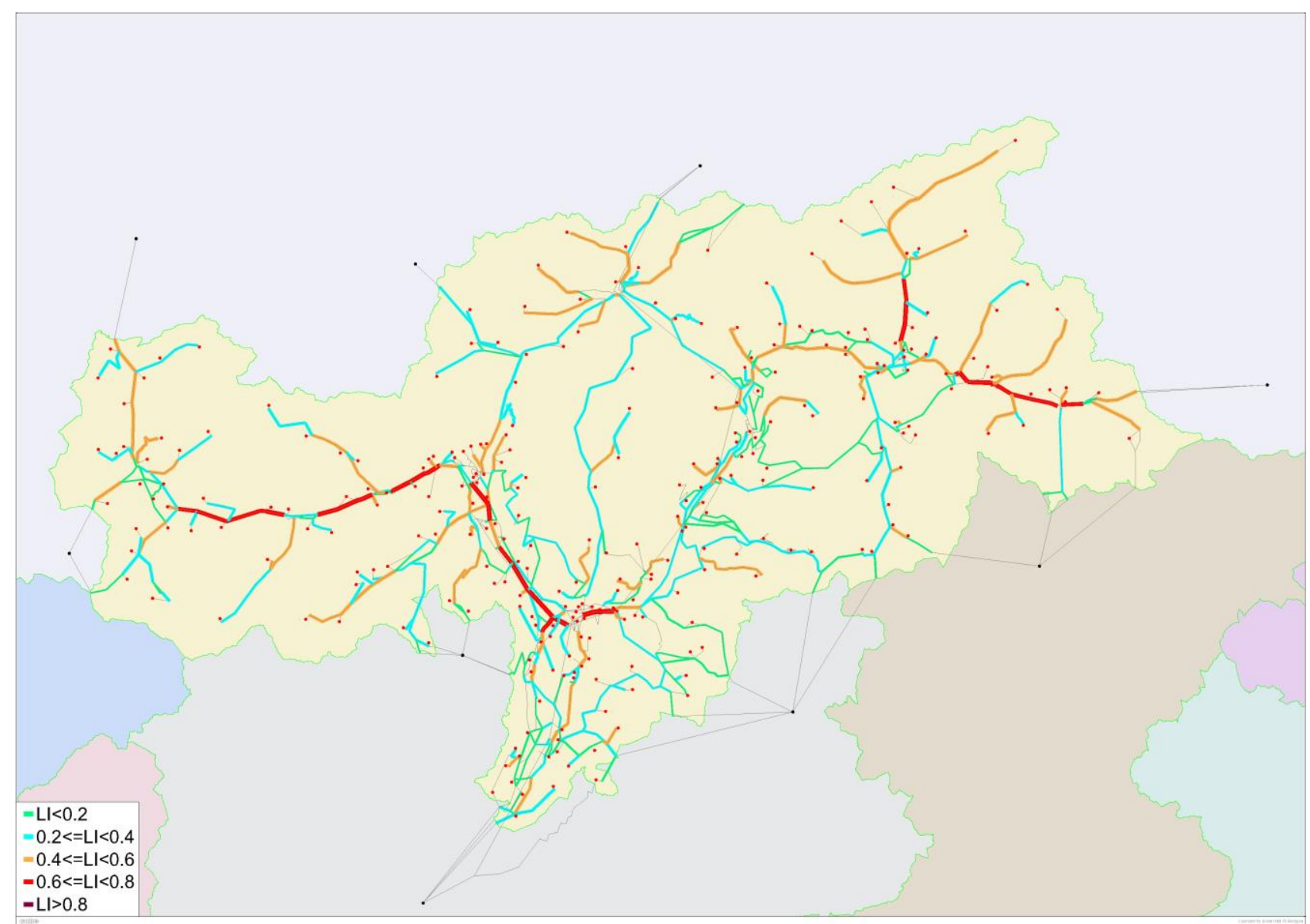

Figure 3 - Link importance index for the network of Bolzano. 
Table 2. Top 20 important links of the road network.

\begin{tabular}{|c|c|c|c|c|c|c|}
\hline Link code & Road name & $\begin{array}{c}A D T_{j} \\
\text { (veh/day) }\end{array}$ & $\begin{array}{c}D_{O D}^{j} \\
\text { (veh/hour) }\end{array}$ & $\begin{array}{l}\text { Rank based } \\
\quad \text { on } F_{j}\end{array}$ & $\begin{array}{l}\text { Rank based } \\
\quad \text { on } G_{j}\end{array}$ & $\begin{array}{l}\text { Rank based } \\
\text { on } L I_{j}\end{array}$ \\
\hline 841 & SS38 & 17,000 & 1,194 & 46 & 3 & 1 \\
\hline 836 & SS38 & 16,214 & 1,123 & 55 & 4 & 2 \\
\hline 1002 & SS621 & 16,560 & 975 & 51 & 5 & 3 \\
\hline 797 & SS12 & 34,099 & 0 & 2 & 181 & 4 \\
\hline 881 & SS42 & 25,000 & 0 & 11 & 182 & 5 \\
\hline 879 & SS42 & 23,593 & 0 & 12 & 186 & 6 \\
\hline 846 & SS38 & 26,292 & 0 & 10 & 193 & 7 \\
\hline 742 & SS12 & 34,099 & 0 & 3 & 197 & 8 \\
\hline 851 & SS38 & 30,544 & 0 & 5 & 199 & 9 \\
\hline 852 & SS38 & 35,913 & 0 & 1 & 203 & 10 \\
\hline 741 & SS12 & 34,099 & 0 & 4 & 208 & 11 \\
\hline 877 & SS42 & 21,928 & 0 & 13 & 210 & 12 \\
\hline 875 & SS42 & 20,172 & 0 & 17 & 190 & 13 \\
\hline 876 & SS42 & 21,282 & 0 & 15 & 217 & 14 \\
\hline 847 & SS38 & 30,500 & 0 & 6 & 218 & 15 \\
\hline 942 & SS49 & 15,000 & 798 & 63 & 8 & 16 \\
\hline 848 & SS38 & 30,500 & 0 & 8 & 250 & 17 \\
\hline 834 & SS38 & 14,500 & 936 & 67 & 6 & 18 \\
\hline 629 & $\mathrm{SS} 238$ & 20,000 & 0 & 19 & 240 & 19 \\
\hline 850 & SS38 & 12,406 & 0 & 9 & 317 & 20 \\
\hline
\end{tabular}

Figure 3 visualizes the results on the network, and Table 2 reports the top 20 links in the final link importance ranking - with a comparison with the different ranking obtained considering either the local or the global importance. The most critical link of the network is part of the national road SS38, with an ADT of 17,000 veh/day and an unassigned demand of $1194 \mathrm{veh} /$ hour estimated in case the link is interrupted. Other links belonging the same road SS38 follow in the ranking. Although these links do not have the highest $A D T$, they are located on roads with relatively high traffic volumes where there are no alternatives routes. Thus, if closed, part of the demand remain unassigned, hence they present high values of global importance index. Other particularly important links are those on the national roads SS621, SS12 and SS42. It is worth noting that the top 3 links in the ranking are cut links. Other cut links, with lower ADT values, follow next in the ranking. Clearly, this is a result of combining local and global importance to determine link importance. 
To better compare the different rankings in their entirety, Spearman's rank correlation coefficient $\rho$ is calculated (Spearman, 1904). If comparing the rankings based on $L I_{j}$ and $F_{j}, \rho=0.42$, while if comparing the rankings based on $L I_{j}$ and $G_{j}, \rho=0.77$. This means that results noticeably changes when considering the different importance indexes. Significantly, highly congested links usually have alternative routes in case they are interrupted. As a consequence, although their local importance is high, the increase in generalised trip cost if these links get interrupted is often not as high as that resulting from the closure of less congested cut links.

A sensitivity analysis has been performed regarding the importance index $L I$, with reference to variations in the value of parameter $\beta$. Indeed, parameter $\beta$ has been gradually increased from 0 to 1. Then, for each value of $\beta$ chosen within such an interval, the values of the importance index $L I$ have been computed, which correspond to all the links under study. In all cases, importance ranks have been obtained by listing links according to a decreasing order of their $L I$ value. For each value of $\beta$ and in comparison to the base case $\beta=0$, the overall variation of link ranks has been measured by means of Spearman's rank correlation coefficient. The results of such comparisons are represented in Figure 4.

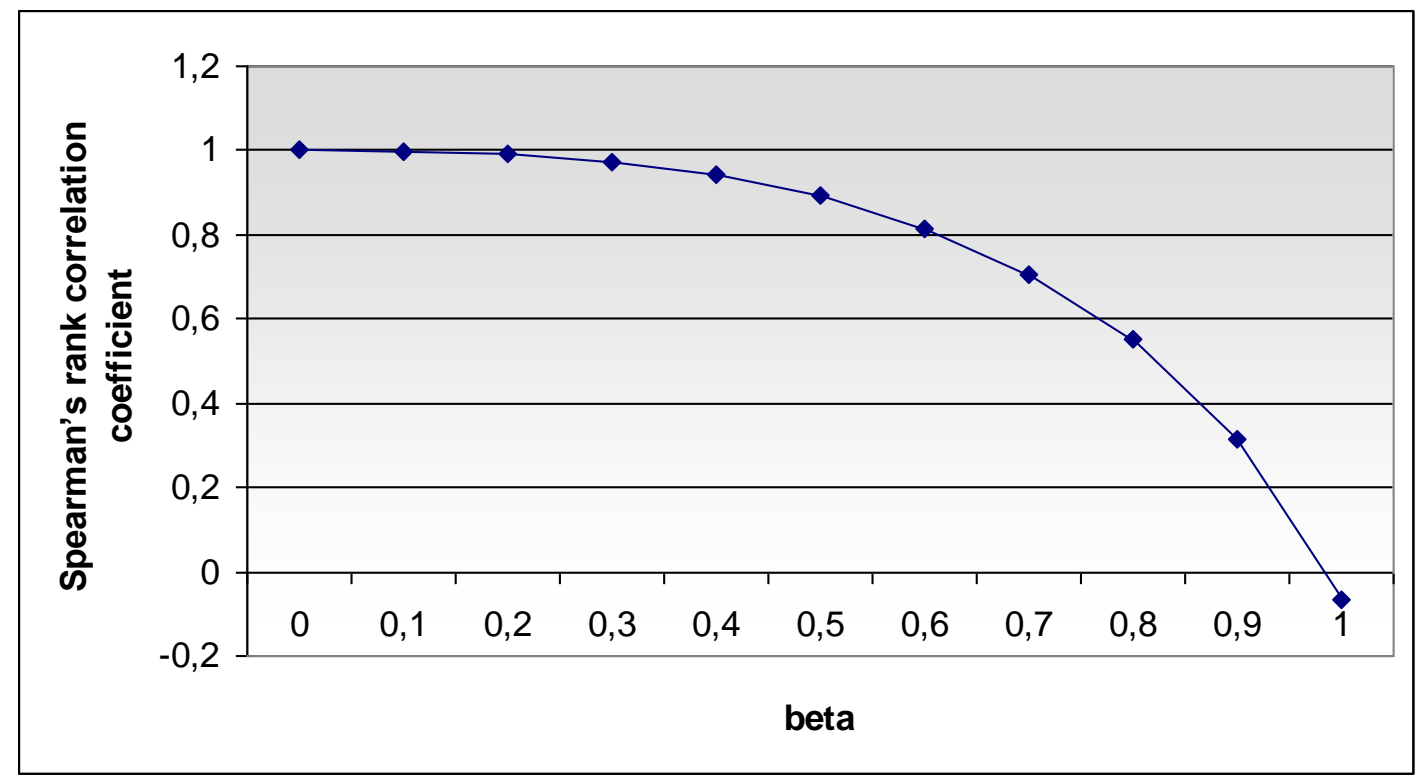

Fig. 4. Sensitivity analysis varying parameter $\beta$

Figure 4 displays how the link rankings vary with the increase of $\beta$ parameter's value (i.e., from $\beta=0$ to $\beta=0.5$ the Spearman's coefficient is reduced by about $11 \%$ ). 


\section{Conclusions}

The analysis of a road network in terms of its vulnerability is illustrated in this paper, examining the case of a mountain area in the Italian Alps, with potential for reproducing the application to any road network whose operations may be jeopardised by natural hazards or external factors.

The methodology implemented in this research estimates the vulnerability of a road network by estimating the vulnerability of its individual links, that is assumed to be proportional to their importance in providing transport connections between each $O-D$ pair. Even without directly considering the problem of the probability of a link disruption - which involves specific studies on the disrupting events in the area under examination -, a review of the literature points to a great variety in the definition of vulnerability and how it can be measured in real-scale networks. In this paper, we consider vulnerability to be the importance of a network's link with respect to maintaining the network functionality.

In the present study we introduced a new methodology which considers that the importance of a link is composed of two aspects: its level of usage, i.e. how many people typically travel along the link, and the impact that closing the link can have on the general functioning of the network as a whole. The first aspect, which we call local importance, requires a measure based on the average daily traffic $(A D T)$. The second aspect, which we call global importance, is evaluated as a function of the variation in the total-trip cost $\left(\Delta C_{j},\right)$, comparing the undamaged with the damaged scenario, where the link $j$, whose importance is to be determined, is assumed to be completely closed.

In addition, in order to account for the presence of cut links - i.e. those links which, if closed, determine a disconnection between two parts of the network - a special formulation for global importance measure was defined, based upon the unassigned demand resulting from the closure of a cut link.

The implementation of the procedure illustrated in this paper allow to obtain a ranking in decreasing order of importance for the links of the network; such a ranking includes all links being examined, both the cut links and the non-cut links.

The methodology was tested on the real-scale road network system in the area of Bolzano, which is a highly mountainous area located in the Alps of northern Italy. The topology of this network is defined by a significant presence of cut links. The application of the methodology led to satisfactory results, in terms of ranking the links in decreasing order depending on their importance scores.

The results obtained can easily be used by practitioners and decision-makers and are relevant, for instance, in determining a list of priorities in the allocation and/or orientation of economic resource for infrastructure maintenance and improvement work. 


\section{Acknowledgements}

This research has been co-financed in the framework of the EU PARAmount project. Authors are very grateful to Ms Claudia Strada, Mr. Matteo Mottironi and all the staff of Provincia Autonoma di Bolzano for their input data, information for the model calibration and the kind collaboration they provided in all phases of the research: without their support this paper could not have been written.

\section{References}

- Bell, M.G.H. (2000), “A game theory approach to measuring the performance reliability of transportation networks", Transportation Research Part B, vol. 34 (6), pp. 533-545.

- Berdica K. (2002), "An introduction to road vulnerability: what has been done, is done and should be done", Transport Policy, vol.9, pp. 117-127.

- Berdica, K. and Mattsson, L. G. (2007) "Vulnerability: a model-based case study of the road network in Stockholm", Critical Infrastructure, pp. 81-106.

- Bono, F., Gutiérrez, E. (2011), “A network-based analysis of the impact of structural damage on urban accessibility following a disaster: the case of the seismically damaged Port Au Prince and Carrefour urban road networks", Journal of Transport Geography, vol.19 (6), pp. 1443-1455.

- Bureau of Public Roads (1964), Traffic Assignment Manual, Urban Planning Division, U.S. Department of Commerce, Washington, DC.

- Carlson, L., Bassett, G., Buehring, W., Collins, M., Folga, S., Haffenden, B., Petit, F., Phillips, J., Verner, D. and Whitfield, R. (2012) Resilience: theory and applications. Argonne National Laboratory. Argonne, Illinois, USA. Retrieved from: http://www.dis.anl.gov/pubs/72218.pdf

- Cascetta E. (2008), “Transportation Systems Analysis: Models and Applications”, SpringerVerlag, New York Inc. 
- Chen, A., Yang, C., Kongsomsaksakul, S. and Lee, M. (2007), "Network-based accessibility measures for vulnerability analysis of degradable transportation networks", Networks and Spatial Economics vol.7 (3), pp. 241-256.

- D’Este, G.M. and Taylor, M.A.P. (2003), "Network vulnerability: an approach to reliability analysis at the level of national strategic transport networks", Iida, Y., Bell, M.G.H. (Eds.), The Network Reliability of Transport, pp. 23-44.

- Du, L. and Peeta, S. (2014), “A Stochastic Optimization Model to Reduce Expected PostDisaster Response Time Through Pre-Disaster Investment Decisions", Networks and Spatial Economics, pp. 1-25.

- Du, Z.P. and Nicholson, A. (1997), "Degradable transportation systems: Sensitivity and reliability analysis”, Transportation Research Part B, vol. 31(3), pp. 225-237.

- Husdal, J. (2004), "Reliability and vulnerability versus cost and benefits", Proceedings of the second International Symposium on Transportation Network Reliability (INSTR), Christchurch and Queenstown, New Zealand.

- Immers, L.H., Stada, J.E., Yperman, I. (2004), "Robustness and resilience of transportation networks; problem survey and examples". Paper Presented at NECTAR Cluster Meeting on Reliability of Networks, Amsterdam, March 19-20.

- Jenelius, E., Petersen, T. and Mattsson, L.G. (2006), "Importance and exposure in road network vulnerability analysis", Transportation Research Part A, vol. 40, n.7, pp. 537-560.

- Jenelius, E. (2009), "Network structure and travel patterns: Explaining the geographical disparities of road network vulnerability", Journal of Transport Geography, vol. 17(3), pp. 234-244.

- Jenelius, E. (2010), "Redundancy importance: Links as rerouting alternatives during road network disruptions", 1st International Conference on Evacuation Modelling and Management, pp. 129-137.

- Knoop, V., van Zuylen, H. and Hoogendoorn, S. (2008), "The influence of spillback modelling when assessing consequences of blockings in a road network", European Journal of Transport and Infrastructure Research, vol. 8(4), pp. 287-300.

- Luathep, P., Sumalee, A., Ho, H. W., Kurauchi, F. (2011), "Large-scale road network vulnerability analysis: a sensitivity analysis based approach", Transportation, vol. 38(5), pp. 799-817.

- Matisziw, T. C., Murray, A. T., Grubesic, T. H. (2010), "Strategic Network Restoration", Networks and Spatial Economics, vol. 10 (3), pp. 345-361. 
- Qiang, Q. and Nagurney, A. (2008), “A unified network performance measure with importance identification and the ranking of network components", Optimization Letters 2, pp. 127-142.

- Reggiani, A., Nijkamp, P. and Lanzi, D. (2014), “Transport resilience and vulnerability: the role of connectivity", Transportation Research A (forthcoming)

- Rose, A. (2009) Economic resilience to disasters. CARRI Report No. 8, Community and Resilience Institute. Retrieved from:

http://www.resilientus.org/library/Research_Report_8_Rose_1258138606.pdf

- Sarewitz , D., Pielke, R.J. and Keykhah, M. (2003), "Vulnerability and risk: some thoughts from a political and policy perspective", Risk Analysis, vol. 23(4), pp. 805-810.

- Scott, D. M., Novak, D. C., Aultman-Hall, L., Guo, F. (2006), "Network Robustness Index: A new method for identifying critical links and evaluating the performance of transportation networks", Journal of Transport Geography, vol. 14(3), pp.215-227.

- Snelder, M., van Zuylen, H.J. and Immers, L.H. (2012), “A framework for robustness analysis of road networks for short term variations in supply", Transportation Research Part A, vol. 46 (5), pp. 828-842.

- Spearman, C. (1904), "The proof and measurement of association between two things", The American Journal of Psychology, vol. 15(1), pp. 72-101.

- Sumalee, A., Kurauchi, F. (2006), "Network Capacity Reliability Analysis Considering Traffic Regulation after a Major Disaster", Networks and Spatial Economics, vol. 6(3-4), pp. 205-219.

- Sullivan, J.L., Novak, D.C., Aultman-Hall, L. and Scott, D.M. (2010), "Identifying critical road segments and measuring system-wide robustness in transportation networks with isolating links: a link-based capacity-reduction approach", Transportation Research Part A, vol. 44, pp. 323-336.

- Taylor, M. A. P., Sekhar, S. V. C., D’Este, G. M. (2006), “Application of Accessibility Based Methods for Vulnerability Analysis of Strategic Road Networks", Networks and Spatial Economics, vol. 6(3-4), pp. 267-291.

- Taylor, M. A.P. and Susilawati, S. (2012), "Remoteness and accessibility in the vulnerability analysis of regional road networks", Transportation Research Part A, vol. 46, pp. 761-771.

- Yin, Y., Madanat, S. M., Lu, X. Y. (2009). "Robust improvement schemes for road networks under demand uncertainty", European Journal of Operational Research, vol. 2(198), pp. 470-479. 